BULLETIN OF THE

AMERICAN MATHEMATICAL SOCIETY

Volume 80, Number 3, May 1974

\title{
HARMONIC QUASICONFORMAL MAPPINGS OF RIEMANNIAN MANIFOLDS
}

\author{
BY SAMUEL I. GOLDBERG ${ }^{1}$ AND TORU ISHIHARA
}

Communicated by S. S. Chern, August 1, 1973

1. Introduction. In this note, we announce some results concerning the distance-volume-decreasing property of harmonic quasiconformal mappings of Riemannian manifolds. Details will appear elsewhere.

Let $M$ and $N$ be $C^{\infty}$ Riemannian manifolds of dimensions $m$ and $n$, respectively. Let $f: M \rightarrow N$ be a $C^{\infty}$ mapping. The Riemannian metrics of $M$ and $N$ can be written locally as $d s_{M}^{2}=\omega_{1}^{2}+\cdots+\omega_{m}^{2}$ and $d s_{N}^{2}=\omega_{1}^{* 2}+$ $\cdots+\omega_{n}^{* 2}$, where $\omega_{i}(1 \leqq i \leqq m)$ and $\omega_{a}^{*}(1 \leqq a \leqq n)$ are linear differential forms in $M$ and $N$, respectively. The structure equations in $M$ are

$$
\begin{gathered}
d \omega_{i}=\sum_{j} \omega_{j} \wedge \omega_{j i}, \\
d \omega_{i j}=\sum_{j} \omega_{i k} \wedge \omega_{k j}-\frac{1}{2} \sum_{k, l} R_{i j k l} \omega_{k} \wedge \omega_{l} .
\end{gathered}
$$

Similar equations are valid in $N$ and we will denote the corresponding quantities in the same notation with asterisks. Let $f^{*} \omega_{a}^{*}=\sum_{i} A_{i}^{a} \omega_{i}$. Then the covariant differential of $A_{i}^{a}$ is defined by

$$
D A_{i}^{a} \equiv d A_{i}^{a}+\sum_{j} A_{j}^{a} \omega_{j i}+\sum_{b} A_{\imath}^{b} \omega_{b a}^{*} \equiv \sum_{j} A_{i j}^{a} \omega_{j}
$$

with $A_{i j}^{a}=A_{j i}^{a}$. The mapping $f$ is called harmonic (resp. totally geodesic) if $\sum_{i} A_{i i}^{a}=0$ (resp. $A_{i j}^{a}=0$ ).

If $m=n$, then at each point of $M$ the matrix $\left(A_{i}^{a}\right)$ has the adjoint $\left(B_{a}^{i}\right)$. Let $C$ be the scalar invariant $\sum B_{a}^{i} B_{b}^{k} A_{k j}^{a} A_{i j}^{b}$. In [2], Chern and one of the authors proved the following theorems which may be regarded as extensions of Schwarz's lemma.

THEOREM I. Let $B^{n}$ be the n-dimensional open ball with the standard hyperbolic metric and $N$ an n-dimensional Riemannian manifold. Let $f: B^{n} \rightarrow N$ be a harmonic mapping satisfying the condition $C \leqq 0$. If $N$ is an Einstein manifold with scalar curvature $R^{*} \leqq-4 n(n-1)$ or if the sectional curvature of $N$ is $\leqq-4$, then $f$ is volume-decreasing.

AMS (MOS) subject classifications (1970). Primary 53A99, 53C99, 30A60.

Key words and phrases. Harmonic mappings, quasiconformal mappings, Schwarz's lemma, distance-volume decreasing.

${ }^{1}$ This research was supported in part by the National Science Foundation. Science Research Council Professor at the University of Liverpool. 
THEOREM II. Let $E^{n}$ be the $n$-dimensional euclidean space and let $N$ be a Riemannian manifold of the same dimension. Let $f: E^{n} \rightarrow N$ be a harmonic mapping satisfying the condition $C \leqq 0$. If $N$ is an Einstein manifold with negative scalar curvature which is bounded away from zero or if the sectional curvature of $N$ is negative and bounded away from zero, then $f$ is volumedecreasing.

The differential $f_{*}$ of $f$ is extended to the mapping $\bigwedge^{p} f_{*}: \bigwedge^{p} T(M) \rightarrow$ $\bigwedge^{p} T(N)$, i.e. the $p$ th exterior power of $f_{*} . \bigwedge^{p} f_{*}$ is also regarded as an element of $\bigwedge^{p} T^{*}(M) \otimes \bigwedge^{p} T(N)$ on which a norm is defined in terms of the Riemannian metrics of $M$ and $N$. The norm $\left\|\wedge^{p} f_{*}\right\|$ is regarded as the ratio function of intermediate volume elements of $M$ and $N$ [7]. In particular, $\left\|\wedge^{1} f_{*}\right\|=\left\|f_{*}\right\|$ may be considered as the ratio of distances. When $m=n,\left\|\wedge^{n} f_{*}\right\|$ is the ratio of volume elements.

The Laplacian of $\left\|\wedge^{n} f_{*}\right\|$ in the case $m=n$ plays an important role in [2]. In this paper, we apply the Laplacian $\Delta$ to $\left\|f_{*}\right\|^{2}$ and obtain the following formula when $f$ is harmonic.

$$
(1 / 2) \Delta\left\|f_{*}\right\|^{2}=\sum_{a, i, j}\left(A_{i j}^{a}\right)^{2}+\sum_{a, i, j} R_{i j} A_{i}^{a} A_{j}^{a}-\sum_{a, b, c, d ; i, j} R_{a b c d}^{*} A_{i}^{a} A_{j}^{b} A_{i}^{c} A_{j}^{d},
$$

where $R_{i j}$ is the Ricci tensor of $M$ (see also [3], [4]). This formula leads to several extensions of Schwarz's lemma as well as a generalization of Liouville's theorem and the little Picard theorem.

2. Quasiconformal mappings. At each point $x \in M$, let $A$ be the matrix representation of $\left(f_{*}\right)_{x}$ relative to orthonormal bases of $T_{x}(M)$ and $T_{f(x)}(N)$ and let ${ }^{t} A$ be the transpose of $A$. In the sequel, we assume rank $f_{*}=$ rank $A=k$ at every point. Then $k \leqq \min (m, n)$ and rank $G=k$, where $G$ is the positive semidefinite symmetric matrix ${ }^{t} A A$. Let $\lambda_{1} \geqq \cdots \geqq \lambda_{k}>$ $\lambda_{k+1}=\cdots=\lambda_{m}=0$ be the eigenvalues of $G$. The norm $\left\|\wedge^{p} f_{*}\right\|$ is represented as

$$
\left\|\wedge^{p} f_{*}\right\|^{2}=\sum_{i_{1}<\cdots<i_{p}}^{k} \lambda_{i_{1}} \cdots \lambda_{i_{p}}
$$

Hence, from Newton's inequalities, we obtain

LEMMA 1. Let $k \leqq \min (m, n)$ and suppose $\operatorname{rank} f_{*}$ is $k$ everywhere on $M$. Then,

$$
\left(\left\|\wedge^{p} f_{*}\right\|^{2} /\left(\begin{array}{l}
k \\
p
\end{array}\right)\right)^{1 / p} \geqq\left(\left\|\wedge^{q} f_{*}\right\|^{2} /\left(\begin{array}{l}
k \\
q
\end{array}\right)\right)^{1 / q}, \quad 1 \leqq p \leqq q \leqq k .
$$

The notion of a $K$-quasiconformal mapping of Riemannian manifolds is now extended to manifolds of different dimensions. (This should result in an extension of Wu's work on normal families of holomorphic mappings 
[8].) At each point $x \in M$, let $S^{k-1}$ be a unit $(k-1)$-sphere in $T_{x}(M)$. If $\left(f_{*}\right)_{x}$ has maximal rank $k$, that is, if $\operatorname{rank}\left(f_{*}\right)_{x}=k=\min (m, n)$, the image of $S^{k-1}$ under $\left(f_{*}\right)_{x}$ is an ellipsoid of dimension $k-1$.

Definition. Let $f$ be a $C^{\infty}$ mapping of maximal $\operatorname{rank} k(=\min (m, n))$ and $K \geqq 1 . f$ is $K$-quasiconformal if at each point $x$ of $M$, the ratio of the largest to the smallest axis of the ellipsoid $\left(f_{*}\right)_{x}\left(S^{k-1}\right)$ in $T_{f(x)}(N)$ $\leqq K$.

One may verify that $f$ is $K$-quasiconformal if and only if $\lambda_{1} / \lambda_{k} \leqq K^{2}$ at each point. Hence, from (2) we obtain

Lemma 2. If $f$ is K-quasiconformal, then

$$
\left(\left\|\wedge^{p} f_{*}\right\|^{2} /\left(\begin{array}{l}
k \\
p
\end{array}\right)\right)^{1 / p} \leqq K^{2}\left(\left\|\wedge^{a} f_{*}\right\|^{2} /\left(\begin{array}{l}
k \\
q
\end{array}\right)\right)^{1 / q}, \quad 1 \leqq p<q \leqq k .
$$

3. Statement of results. First, with no assumption on the quasiconformality of $f$, formula (1) yields

Proposition 1. Let $M$ be a compact manifold and $N$ a manifold with nonpositive sectional curvature. Let $f$ be a harmonic mapping of $M$ into $N$. If $M$ is an Einstein manifold with positive scalar curvature $R$, or if the sectional curvature of $M$ is positive, then $f$ is a constant mapping.

Proposition 2. Let the sectional curvature of $N$ be nonpositive and $f$ be a totally geodesic mapping. If $M$ is an Einstein manifold with positive scalar curvature $R$, or if the sectional curvature of $M$ is positive, then $f$ is a constant mapping.

In the case when $M$ and $N$ have the same dimension $n$, then by means of Lemma 2, Theorem I gives

Proposition 3. Under the conditions in Theorem I with $f$ a K-quasiconformal mapping,

In particular,

$$
\left\|\wedge^{p} f_{*}\right\|^{2} \leqq\left(\begin{array}{l}
n \\
p
\end{array}\right) K^{2 p}, \quad 1 \leqq p \leqq n .
$$

$$
f^{*}\left(d s_{N}^{2}\right) \leqq n K^{2} d s_{B}^{2} .
$$

We return now to the case where the dimensions of $M$ and $N$ are $m$ and $n$, respectively.

THEOREM 1. Let $M$ and $N$ be Riemannian manifolds of dimensions $m$ and $n$ respectively. Let $f: M \rightarrow N$ be a harmonic K-quasiconformal mapping with the function $\left\|f_{*}\right\|$ attaining its maximum on $M$. If

(a) the sectional curvature of $M$ is bounded below by a nonpositive constant $-A$, or $M$ is an Einstein manifold with scalar curvature $R \geqq-m(m-1) A$, 
and

(b) the sectional curvature of $N$ is bounded above by a negative constant $-B$, then

$$
\left\|\wedge^{p} f_{*}\right\|^{2 / p} \leqq(m-1 / k-1)\left(\begin{array}{l}
k \\
p
\end{array}\right)^{1 / p}(A / B) K^{4} \quad 1 \leqq p \leqq k,
$$

where $k=\min (m, n)$.

This theorem improves and generalizes the results of Kiernan [5] and one of the authors [4]. The proof proceeds by first taking a maximum point $x$ of $\left\|f_{*}\right\|^{2}$. Then $\Delta_{x}\left\|f_{*}\right\|^{2} \leqq 0$ and formula (1) yield

$$
2\left\|\wedge^{2} f_{*}\right\|_{x}^{2} \leqq(m-1)(A / B)\left\|f_{*}\right\|_{x}^{2} .
$$

From this, together with Lemmas 1 and 2, the results follow.

In the case when $k=m=n$, Theorem 1 implies that $f$ is volume-decreasing provided $B=A K^{4}$. Moreover, for the ratio $\left\|f_{*}\right\|$ of distances we have the following.

COROLlaRY. Under the assumptions in Theorem 1,

$$
\left\|f_{*}(X)\right\|^{2} \leqq(m-1 / k-1) k(A / B) K^{4}\|X\|^{2}
$$

for every tangent vector $X \in T(M)$. If $B=(m-1 / k-1) k A K^{4}$, then $f$ is distance-decreasing.

If in Proposition 3 the assumption that the curvature of $N$ is $\leqq-4$ is replaced by the assumption that the curvature of $N$ is $\leqq-4 K^{4}$ then the condition on the invariant $C$ may be removed.

THEOREM 2. Let $B^{m}$ be the m-dimensional unit open ball with the hyperbolic metric of constant curvature -4 . Let $N$ be a Riemannian manifold with sectional curvature bounded above by a negative constant $-B$. Then, if $f: M \rightarrow N$ is a harmonic K-quasiconformal mapping

where $k=\min (m, n)$.

$$
\left\|\wedge^{p} f_{*}\right\|^{2} \leqq\left[4\left(\frac{m-1}{k-1}\right) \frac{K^{4}}{B}\right]^{p}\left(\begin{array}{l}
k \\
p
\end{array}\right), \quad 1 \leqq p \leqq k,
$$

COROLlary 1. Under the conditions in Theorem 2 with $B=$ $4 k(m-1 / k-1) K^{4}$, the mapping $f$ is distance-decreasing.

COROLlary 2. If in addition to the hypotheses of Theorem $2, \operatorname{dim} N=m$ and $B=4 K^{4}$, the mapping $f$ is volume-decreasing.

REMARK. If $\lambda_{1} \geqq \lambda_{2} \geqq \cdots \geqq \lambda_{k} \geqq \lambda_{k+1} \geqq \cdots \geqq \lambda_{m} \geqq 0$, the above results are still valid if the condition that $f$ be $K$-quasiconformal is replaced by the condition (Q): At each point $x$ of $M, \lambda_{1} \leqq K^{2} \lambda_{k}$ where $K \geqq 1$ is a given constant and $k=\min (m, n)$. 
DEFINITION. A smooth mapping $f$ of an $m$-dimensional Riemannian manifold $M$ into an $n$-dimensional Riemannian manifold $N$ satisfying the condition (Q) is called a $K$-quasiconformal mapping in the generalized sense.

Regarding Theorem II, the technique employed in establishing Theorem 2 also yields

THEOREM 3. Let $N$ be an n-dimensional Riemannian manifold with negative sectional curvature bounded away from zero, and let $f: E^{m} \rightarrow N$ be a harmonic $K$-quasiconformal mapping in the generalized sense. Then, $f$ is a constant mapping.

The classical theorem of Liouville states that every bounded holomorphic function on the entire complex plane $C$ is a constant. On the other hand, an entire function with two lacunary values must be a constant. This is the little Picard theorem. Theorem 3 generalizes Liouville's theorem as well as the little Picard theorem, the latter case being a consequence of the fact that the Gaussian plane minus two points carries a Kaehler metric of constant negative curvature.

\section{BIBLIOGRAPHY}

1. S. S. Chern, On holomorphic mappings of hermitian manifolds of the same dimension, Entire Functions and Related Parts of Analysis (Proc. Sympos. Pure Math., La Jolla, Calif., 1966), Amer. Math. Soc., Providence, R.I., 1968, pp. 157-170. MR 38 \#2714.

2. S. S. Chern and S. I. Goldberg, On the volume-decreasing property of a class of real harmonic mappings, Amer. J. Math. (to appear).

3. J. Eells Jr. and J. H. Sampson, Harmonic mappings of Riemannian manifolds, Amer. J. Math. 86 (1964), 109-160. MR 29 \#1603.

4. T. Ishihara, A remark on harmonic quasiconformal mappings, J. Math. Tokushima Univ. 5 (1971), 17-23. MR 46 \#829.

5. P. J. Kiernan, Quasiconformal mappings and Schwarz'slemma, Trans. Amer. Math. Soc. 148 (1970), 185-197. MR 41 \#467.

6. S. Kobayashi, Volume elements, homomorphic mappings and Schwarz's lemma, Entire Functions and Related Parts of Analysis (Proc. Sympos. Pure Math., La Jolla, Calif., 1966), Amer. Math. Soc., Providence, R.I., 1968, pp. 253-260. MR 38 \#6100.

7. Y. C. Lu, Holomorphic mappings of complex manifolds, J. Differential Geometry 2 (1968), 299-312. MR 40 \#3482.

8. H. Wu, Normal families of holomorphic mappings, Acta Math. 119 (1967), 193-233. MR 37 \#468.

Department of Mathematics, University of Illinois, Urbana, Illinois

Department of Mathematics, University of LiVerpool, Liverpool, ENGLAND

Department of Mathematics, Tokushima University, Josanjima, Japan 